\title{
LESÕES CARDÍACAS EM RATOS WISTAR INOCULADOS COM DIFERENTES CEPAS DO TRYPANOSOMA CRUZI
}

\author{
E. Chapadeiro, P.S.S. Beraldo, P.C. Jesus, W.P. Oliveira Jr. e \\ L.F. Junqueira Jr.
}

\begin{abstract}
Ratos albinos Wistar infectados com diferentes inóculos das cepas $Y$, Colombiana (COL) e São Felipe (12SF) do Trypanosoma cruzi desenvolveram parasitemia elevada entre 14 e 21 dias, a qual tornou-se desprezivel ou desapareceu completamente ao final da 4a semana.

As lesões do coração foram avaliadas, semiquantitativamente, pelo sistema de "scores", conforme a intensidade e a extensão da inflamação, nas diferentes estruturas do órgão, tendo sido observado que: a) na fase inicial (aguda) da infecção, os animais mortos espontaneamente ou em conseqüência de acidente anestésico apresentaram cardite de intensidade moderada à acentuada, não havendo diferença em relação às cepas utilizadas; as diferentes estruturas do coração estavam uniformemente comprometidas; b) na fase crônica da infecção observou-se cardite de grau leve a moderado e as lesões foram mais freqüentes e intensas quanto maior o inóculo empregado; no entanto, inóculos baixos só produziram lesões quando a cepa utilizada foi a Colombiana. Trombose mural nos atrios e dilatação da ponta do ventriculo esquerdo foram observadas em $20 \%$ dos animais, independentemente da cepa. Na fase crônica $15 \%$ dos animais apresentaram miocardite crônica com fibrose, associada à hipertrofia das miocélulas. Os AA confirmam ser o rato albino suscetivel à infecção chagásica e, portanto, um modelo útil no estudo das manifestações cardíacas da Doença de Chagas.
\end{abstract}

Palavras-chaves: Infecção chagásica. Doença de Chagas experimental. Cardiopatia chagásica experimental.

Vários autores ${ }^{1} 268991214161718$ descreveram lesões morfológicas não somente do coração, mas também de outros órgãos de ratos infectados com diferentes cepas do Trypanosoma cruzi. Todavia, esses achados não parecem suficientes ainda para a inclusão do rato entre os modelos experimentais, da Doença de Chagas ${ }^{19}$, visto que segundo alguns ${ }^{356913}$ o rato é altamente resistente à infecção pelo Trypanosoma cruzi e essa tem caráter imprevisivel.

Trabalho recente ${ }^{4}$ mostra que o rato infectado, por diferentes cepas do Trypanosoma cruzi, desenvolve cardite aguda e crônica, sendo útil, particularmente, para o estudo das alterações autonômicas parassimpáticas e eletrocardiográficas.

Trabalho dos Laboratórios Cardiovascular e Patologia da Faculdade de Cièncias da Saúde da Universidade de Brasilia e do Curso de Pós-Graduação em Patologia Humana da Faculdade de Medicina do Triângulo Mineiro.

Suporte financeiro do Conselho Nacional de Desenvolvimento Científico e Tecnológico - CNPq (Processos n.: 40.3748/82, 100.458/84, 134.168/85).

Endereço para correspondência: Prof. Edmundo Chapadeiro-Coordenadoria de Pós-Graduação-Faculdade de Medicina do Triângulo Mineiro. Praça Thomaz Ulhoa, 706 38025 - Uberaba, MG, Brasil.

Recebido para publicação em 8/7/88.
Este presente trabalho visa relatar, em detalhe, os achados morfológicos dos corações, desses mesmos animais $^{4}$, visto serem escassos os dados da literatura, especialmente no que se relaciona às alterações morfológicas do coração do rato na fase tardia da infecção.

\section{MATERIAL E MÉTODOS}

\section{MATERIAL}

Duzentos e dezessete ratos albinos Wistar $^{4}$, com idade entre 25 e 30 dias, endocriados e fornecidos pelo Biotério Central da Universidade de Brasilia, foram separados aleatoriamente em 4 grupos (Y, SF, COL e Controle). No grupo Y, 59 animais, divididos nos subgrupos A, B e C, foram inoculados, respectivamente, com 2000 a 4000,4000 a 5000 e 6000 formas sangüícolas por grama de peso da cepa $\mathrm{Y}$ do Trypanosoma cruzi; no grupo SF, 45 ratos foram inoculados com a cepa $12 \mathrm{SF}$ nas mesmas condições anteriores e no grupo COL, 73 animais foram inoculados em condições idênticas aos grupos anteriores com a cepa Colombiana; no grupo controle, $\mathbf{4 0}$ ratos, não infectados, serviram de controle ao longo do experimento. 


\section{MÉTODOS}

Ao final do 8 . mês após a infecção, tanto os animais sobreviventes, como aqueles que morreram natural ou acidentalmente - durante os testes da função autonômica e da obtenção do traçado eletrocardiográfico, foram necropsiados, juntamente com os controles da mesma idade. Após a retirada do coração, este era seccionado sagitalmente e colocado in totum em formol neutro a $10 \%$ para o estudo histológico rotineiro e seriado (10 corações de cada grupo) do sistema nervoso intracardiaco e de condução. Fragmentos, das demais vísceras, foram fixados em condições idênticas para estudo posterior.
Para uma acurada correlação, com as alterações eletrocardiográficas e com a função autonômica ${ }^{4}$ foi feita também uma abordagem semiquantitativa das lesões histológicas cardiacas nos 4 grupos, utilizandose o sistema de "scores". Para cada estrutura examinada obteve-se um "score" respectivo; a somatória desses "scores" forneceu um "score total" para cada animal, corrigido para o número de estruturas efetivamente examinadas e denominado "score total corrigido" (Tabela 1).

O teste do qui-quadrado foi utilizado na avaliação da mortalidade produzida pelas três cepas e a análise de variância com teste de Newman-Keuls foi

\section{Tabela 1 - Critérios de avaliaçāo quantitativa das lesões histopatológicas do coraçāo na infecção chagásica experimental no rato.}

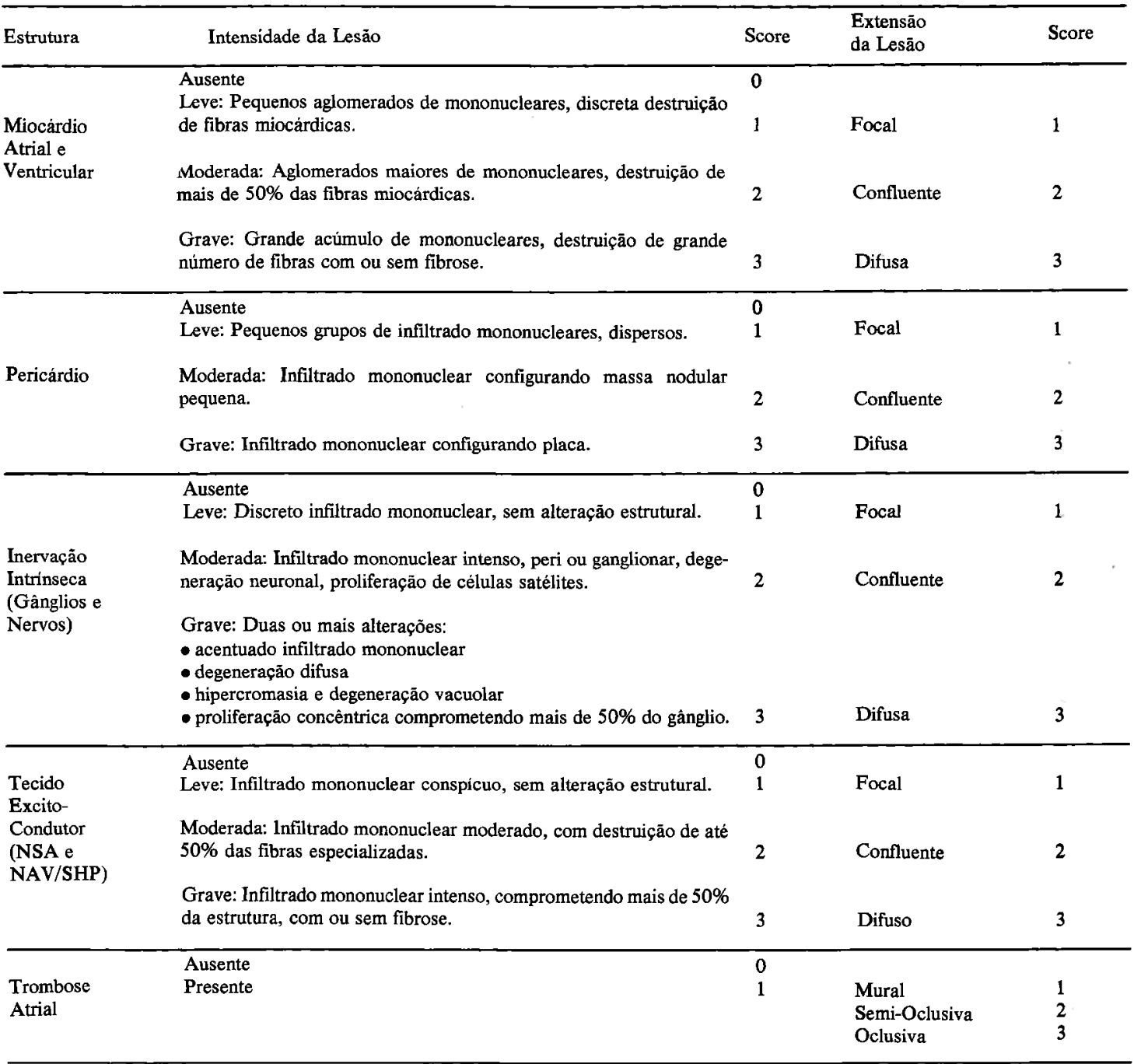


empregada na comparação entre os "scores" encontrados.

\section{RESULTADOS}

\section{INFECÇÃO E MORTALIDADE}

Os animais inoculados desenvolveram parasitemia variável que atingiu o pico máximo entre a $2 \mathrm{a} e$ 3a semanas, declinando progressivamente até o final da 4: semana (fase aguda ou inicial da infecção). Após esse periodo considerou-se a infecção como tardia ou crônica.

A Tabela 2 mostra a mortalidade, espontânea e acidental, pelo anestésico dos ratos inoculados e controles nas fases aguda e crônica (até o $8 \circ$ mês) da infecção, segundo a cepa e o valor do inóculo.

\section{ACHADOS MORFOLÓGICOS DO CORAÇÃO}

\section{FASE AGUDA DA INFECÇÃO}

Macroscopicamente, os corações, da maioria dos ratos inoculada que morreu ou foi sacrificada até o final da 6a semana, apresentavam-se muito pálidos e menores que aqueles dos controles da mesma idade. Trombose recente dos átrios ocorreu na maioria dos animais inoculada com as cepas $\mathrm{Y}$ e Colombiana; nenhun animal inoculado com a cepa $12 \mathrm{SF}$ mostrou sinais de trombose.

Microscopicamente, as alterações cardiacas são do ponto de vista qualitativo, basicamente, as mesmas nos três grupos chagásicos; quantativamente, porém, mostram diferenças. Devido ao pequeno número de animais em cada subgrupo, não foi possivel estabelecer-se uma correlação entre o inóculo e o grau das lesões. Os três folhetos do coração foram atingidos igualmente; contudo, as lesões são mais intensas no miocárdio dos átrios e septo interatrial. $\mathrm{Em}$ ordem decrescente, foram atingidos o miocárdio do ventrículo esquerdo, do septo interventricular e do ventrículo direito. $\mathrm{Na}$ região da ponta dos ventrículos, o músculo foi menos lesado (Figura 1A).

No miocárdio, como nos demais folhetos, a inflamação tem intensidade variável e é geralmente difusa; mas em alguns ratos, tem caráter multifocal. $O$ infiltrado inflamatório é constituido, em sua maior parte, por mononucleares (macrófagos e linfócitos) e, em menor número, por plasmócitos e granulócitos neutrófilos; os mastócitos que são abundantes, nos

Tabela 2 - Mortalidade natural e, associada à anestesia para a obtenção do eletrocardiograma, observada no grupo de ratos-controles enos grupos e subgrupos de ratos chagásicos infectados com as cepas $Y$, São Felipe (SF) e Colombiana (COL) do T. cruzi, durante as fases inicial (menos de 6 semanas) e tardia (mais de 6 semanas) da infeç̧ão.

\begin{tabular}{|c|c|c|c|c|c|c|c|c|c|}
\hline \multirow{3}{*}{ Grupo } & \multirow{3}{*}{ Inóculo } & \multicolumn{7}{|c|}{ Mortalidade } & \\
\hline & & \multicolumn{3}{|c|}{ Fase Inicial } & \multicolumn{5}{|c|}{ Fase Tardia } \\
\hline & & $\mathbf{N}$ & Natural & Anestesia & Total & $\mathbf{N}$ & Natural & Anestesia & Total - \\
\hline Controle & & 40 & $1(2,5 \%)$ & $1(2,5 \%)$ & $2(5,0 \%)$ & 38 & $3(8,0 \%)$ & $3(8,0 \%)$ & $I(16,0 \%)$ \\
\hline $\mathbf{Y}$ & $\begin{array}{c}\mathrm{A} \\
\mathrm{B} \\
\mathrm{C} \\
\mathrm{A}+\mathrm{B}+\mathrm{C}\end{array}$ & $\begin{array}{l}22 \\
25 \\
12 \\
59\end{array}$ & $\begin{array}{c}0 \\
9(36,0 \%) \\
6(50,0 \%) \\
* 15(25,0 \%)\end{array}$ & $\begin{array}{c}3(14,0 \%) \\
0 \\
2(17,0 \%) \\
* 5(8,0 \%)\end{array}$ & $\begin{array}{r}3(14,0 \%) \\
9(36,0 \%) \\
8(67,0 \%) \\
20(34,0 \%)\end{array}$ & $\begin{array}{r}19 \\
16 \\
4 \\
39\end{array}$ & $\begin{array}{c}1(5,0 \%) \\
0 \\
0 \\
1(3,0 \%)\end{array}$ & $\begin{array}{c}0 \\
1(6,0 \%) \\
0 \\
1(3,0 \%)\end{array}$ & $\begin{array}{c}1(5,0 \%) \\
1(6,0 \%) \\
0 \\
2(6,0 \%)\end{array}$ \\
\hline SF & $\begin{array}{c}\mathrm{A} \\
\mathrm{B} \\
\mathrm{C} \\
\mathrm{A}+\mathrm{B}+\mathrm{C}\end{array}$ & $\begin{array}{l}10 \\
25 \\
10 \\
45\end{array}$ & $\begin{array}{c}0 \\
11(44,0 \%) \\
4(40,0 \%) \\
* 15(33,0 \%)\end{array}$ & $\begin{array}{l}0 \\
0 \\
0 \\
0\end{array}$ & $\begin{array}{r}0 \\
11(44,0 \%) \\
4(40,0 \%) \\
* 15(33,0 \%)\end{array}$ & $\begin{array}{r}10 \\
14 \\
6 \\
30\end{array}$ & $\begin{array}{c}1(10,0 \%) \\
0 \\
0 \\
1(3,0 \%)\end{array}$ & $\begin{array}{c}0 \\
3(21,0 \%) \\
1(17,0 \%) \\
4(13,0 \%)\end{array}$ & $\begin{array}{l}1(10,0 \%) \\
3(21,0 \%) \\
1(17,0 \%) \\
5(17,0 \%)\end{array}$ \\
\hline $\mathrm{COL}$ & $\begin{array}{c}\mathrm{A} \\
\mathrm{B} \\
\mathrm{C} \\
\mathrm{A}+\mathrm{B}+\mathrm{C}\end{array}$ & $\begin{array}{l}12 \\
49 \\
12 \\
73\end{array}$ & $\begin{array}{r}1(8,0 \%) \\
15(31,0 \%) \\
8(67,0 \%) \\
* 24(33,0 \%)\end{array}$ & $\begin{array}{c}0 \\
0 \\
3(25,0 \%) \\
* 3(4,0 \%)\end{array}$ & $\begin{array}{r}1(8,0 \%) \\
15(31,0 \%) \\
11(92,0 \%) \\
* 27(37,0 \%)\end{array}$ & $\begin{array}{r}11 \\
34 \\
1 \\
46\end{array}$ & $\begin{array}{r}2(18,0 \%) \\
1(3,0 \%) \\
1(100,0 \%) \\
4(9,0 \%)\end{array}$ & $\begin{array}{c}0 \\
5(15,0 \%) \\
0 \\
5(11,0 \%)\end{array}$ & $\begin{array}{r}2(18,0 \%) \\
6(18,0 \%) \\
1(100,0 \%) \\
9(20,0 \%)\end{array}$ \\
\hline $\begin{array}{l}\text { Total de } \\
\text { chagásicos }\end{array}$ & & 177 & $* 54(31,0 \%)$ & $* 8(5,0 \%)$ & $62(35,0 \%)$ & 115 & $6(5,0 \%)$ & $10(9,0 \%)$ & $16(14,0 \%)$ \\
\hline
\end{tabular}

$N=$ Número de ratos

$A=$ baixo inóculo $(2-3.000$ parasitos $/ g)$

$\mathrm{B}=$ médio inóculo $(4-5.000$ parasitos $/ \mathrm{g})$

$\mathrm{C}=$ alto inóculo (6.000 parasitos/g)

* $p<0,01$ (chagásicos $x$ controles). Teste do $X^{2}$ 
Chapadeiro E, Beraldo PSS, Jesus PC, Oliveira Jr. WP, Junqueira Jr. LF. Lesōes cardiacas em ratos wistar inoculados com diferentes cepas do Trypanosoma cruzi. Revista da Sociedade Brasileira de Medicina Tropical 21: 95-103, Jul-Set, 1988

controles, faltam ou são escassos entre as células mencionadas. $\mathrm{O}$ infiltrado e o edema inflamatório acumulam-se, principalmente no endomísio, dissociando não somente os feixes das fibras, mas estas entre si. Formas amastigotas do $T$. cruzi no interior das fibras integras e livres no endomísio são em geral abundantes, mas por vezes mostram-se escassas; na maioria dos casos existe estreita correlação entre a intensidade do parasitismo e a resposta inflamatória (Fig. 1B).

Os segmentos de fibras não parasitadas exibem diferentes formas de degeneração (vacuolar, hialina, etc.) e necrose (coagulação, hialina, miocitólise). A trama reticulinica e capilar, em torno das fibras necróticas, apresenta-se em colapso, com aspecto condensado.

Também o tecido específico de condução é atingido pelo processo inflamatório destrutivo; o parasitismo, embora freqüente, é menos abundante que no miocárdio contrátil.

No epicárdio a reação inflamatória acomete, principalmente, o tecido gorduroso e o plexo nervoso cardiaco (gânglios e filetes nervosos). Nesses, o infiltrado tem a mesma constituição que aquele do miocárdio. Nos gânglios e filetes nervosos, ocorrem respectivamente ganglionite e periganglionite, neurite e perineurite; fenômenos degenerativo-necróticos dos neurônios são intensos, com evidente redução numérica dos mesmos (Fig. 1C).

O endocárdio parietal é também acometido por extensão, a partir do miocárdio; o infiltrado inflamatório tem as mesmas características já descritas, porém é particularmente intenso e geralmente difuso nos átrios; o parasitismo é escasso. Essa endocardite associa-se, principalmente nos átrios, a tromboses recentes, de tipo mural ou ocludente, nos grupos $\mathrm{Y}$ e COL.

A quantificação por "score", da intensidade e extensão das alterações microscópicas dos corações, observadas nos diferentes grupos de ratos chagásicos na fase aguda, está na Tabela 3. Os "scores" médios, considerando-se todos os grupos de ratos e as diversas estruturas cardíacas examinadas, variaram de 3,6 士 1,7 a 5,5 $\pm 1,0$, em uma possibilidade máxima igual a 6. Esse resultado expressa o acometimento agudo do coração em grau moderado a acentuado. Por outro

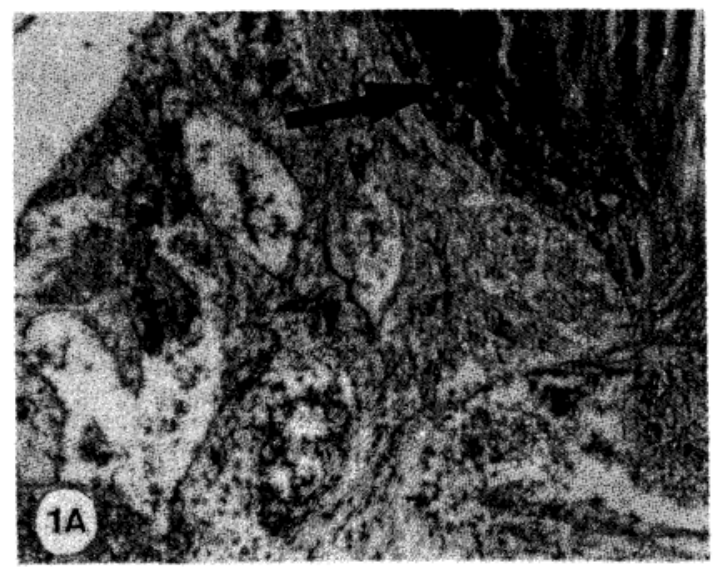

Fig. 1A - Atrio direito. Miocardite e endocardite agudas intensas com trombose recentíssima (seta). Cepa Y. $H E \times 40$.

Fig. 1B - Miocardite aguda com intenso infiltrado de mononucleares e granulócitos entre fibras parasitadas, degeneradas, necrosadas e rótas. Cepa Colombiana. HE $\times 400$.

Fig. 1C - Ganglionite aguda com infiltrado mononuclear. Ninho de amastigotas (seta) em célula satélite. Cepa I2SF, HE $x 400$.
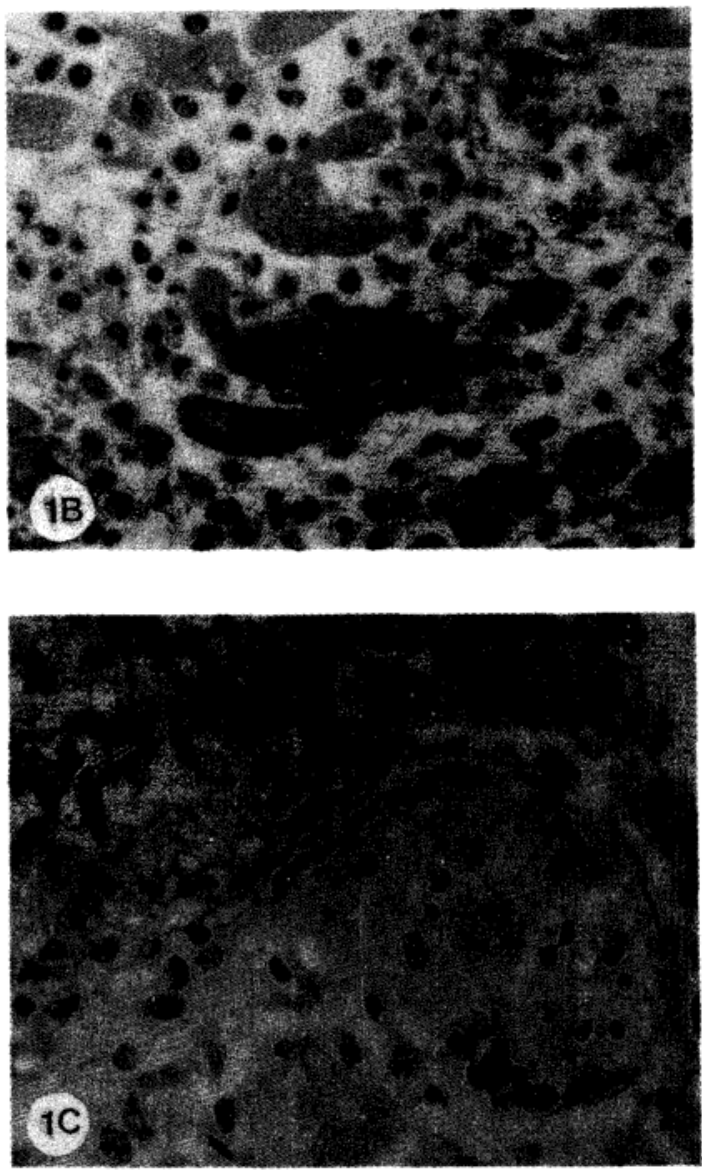
Chapadeiro E, Beraldo PSS, Jesus PC, Oliveira Jr. WP, Junqueira Jr. LF. Lesões cardíacas em ratos wistar inoculados com diferentes cepas do Trypanosoma cruzi. Revista da Sociedade Brasileira de Medicina Tropical 21: 95-103, Jul-Set, 1988

lado, não foram observadas diferenças significativas entre o grupo de ratos chagásicos inoculados com as diferentes cepas, quanto ao grau de lesão de cada estrutura acometida. Não foram também detectadas diferenças significativas entre os graus de acometimento das diversas estruturas cardíacas, observandose grande uniformidade entre as mesmas a esse respeito (Fig. 2).

\section{FASE TARDIA (CRÔNICA) DA INFECÇÃO}

Macroscopicamente, os corações, da maioria dos ratos no 8. mês da infecção, mostram-se semelhantes aqueles dos ratos-controles, sacrificados na mesma época. Em alguns animais, a cavidade do

Tabela 3 - Quantificação por "score", das lesões histopatológicas do coração de ratos chagásicos infectados com as cepas $Y$, Säo Felipe (SF) e Colombiana (COL) do T. cruzi, na fase inicial da infeccão.

\begin{tabular}{|c|c|c|c|c|c|c|c|c|}
\hline \multirow{2}{*}{ Grupo } & \multicolumn{3}{|c|}{ Miocárdio } & \multirow{2}{*}{ Pericárdio } & \multirow{2}{*}{$\begin{array}{l}\text { Inervação } \\
\text { intrinseca }\end{array}$} & \multirow{2}{*}{$\begin{array}{l}\text { Tecido excito- } \\
\text { condutor }\end{array}$} & \multirow{2}{*}{ Trombose } & \multirow{2}{*}{ STC } \\
\hline & $\mathbf{N}$ & Atrial & Ventricular & & & & & \\
\hline $\mathbf{Y}$ & 6 & $\begin{array}{c}4,0 \\
(1,3)\end{array}$ & $\begin{array}{c}4,7 \\
(1,0)\end{array}$ & $\begin{array}{c}4,7 \\
(1,6)\end{array}$ & $\begin{array}{c}5,3 \\
(1,0)\end{array}$ & $\begin{array}{c}5,5 \\
(1,0)\end{array}$ & $\begin{array}{c}1,8 \\
(2,0)\end{array}$ & $\begin{array}{l}0,75 \\
(0,1)\end{array}$ \\
\hline SF & 6 & $\begin{array}{c}4,3 \\
(1,5)\end{array}$ & $\begin{array}{c}4,7 \\
(1,6)\end{array}$ & $\begin{array}{c}4,7 \\
(1,6)\end{array}$ & $\begin{array}{c}4,3 \\
(0,8)\end{array}$ & $\begin{array}{c}4,3 \\
(0,8)\end{array}$ & 0 & $\begin{array}{l}0,66 \\
(0,1)\end{array}$ \\
\hline $\mathrm{COL}$ & 5 & $\begin{array}{c}3,6 \\
(1,7)\end{array}$ & $\begin{array}{c}4,4 \\
(2,2)\end{array}$ & $\begin{array}{c}4,0 \\
(1,4)\end{array}$ & $\begin{array}{c}4,4 \\
(2,2)\end{array}$ & $\begin{array}{c}5,0 \\
(1,7)\end{array}$ & $\begin{array}{c}2,0 \\
(1,9)\end{array}$ & $\begin{array}{l}0,68 \\
(0,3)\end{array}$ \\
\hline
\end{tabular}

- Os valores dados são médias (sd)

- $\mathrm{N}=$ Número de ratos

- STC = "score" total corrigido ("score" total/número de estruturas visualizadas)

- Não houve diferença estatística entre os grupos de ratos ( $>0,05$ - Análise de variância)

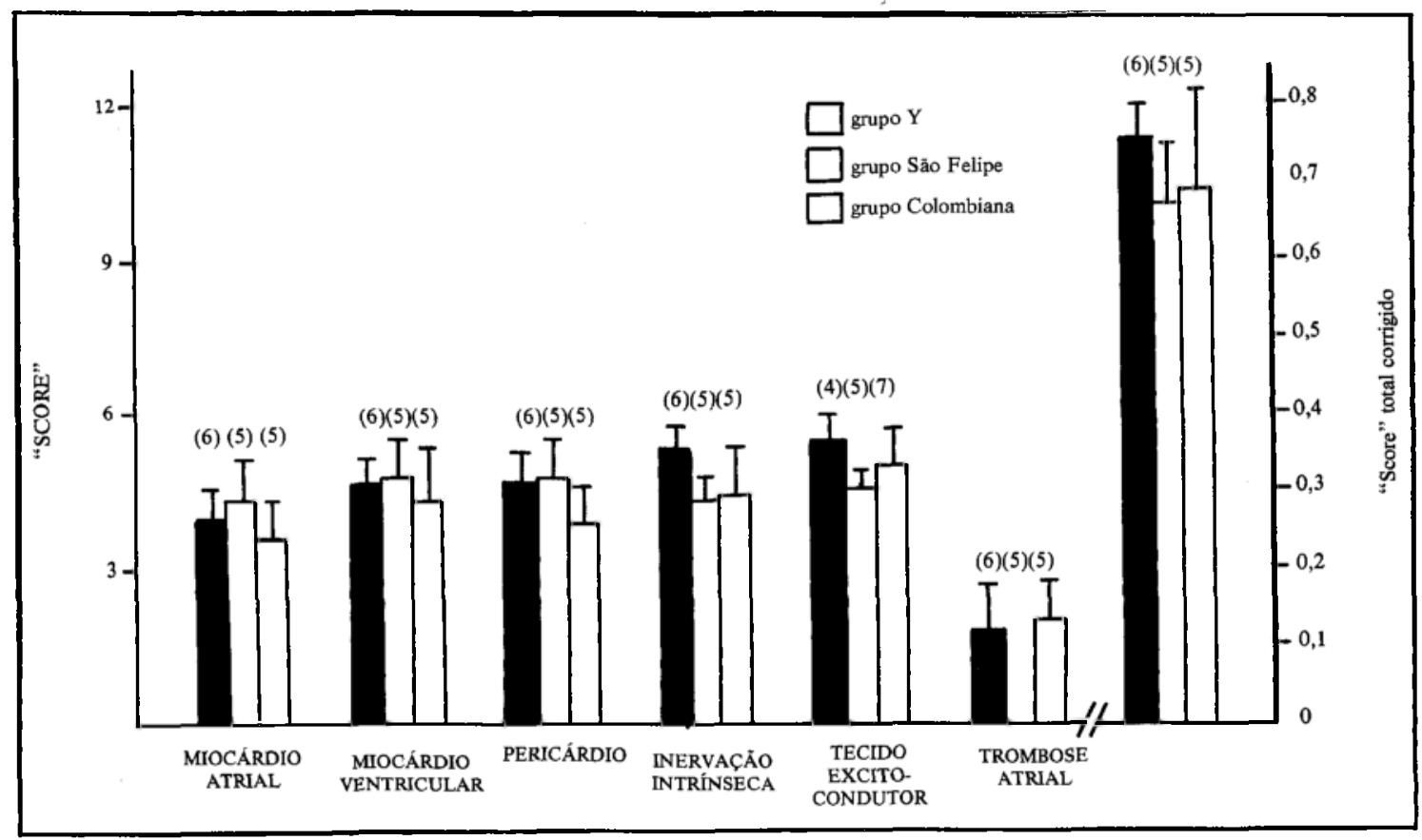

Fig. 2 - Grau de alteraçãohistopatológica, expressa pelo "score"médio e pelo "score" total corrigido, nas diversas estruturas do coração e manifestação de trombose atrial, exibido pelos ratos chagásicos na fase inicial (aguda) da infecção. Não foram observadas diferenças estatisticamente significantes entre os Grupos ( $p>0.05$, análise de variância). Nenhum rato do Grupo São Felipe exibiu trombose atrial, enquanto 3/6(50\%) e 3/5(60\%) dos ratos dos Grupos $Y$ e Colombiana a apresentaram.

( ) Número de ratos nos quais a estrutura foi visualizada. 
ventrículo esquerdo, em correspondência com a ponta, apresentou-se mais dilatada e a parede mais adelgaçada, lembrando a lesão do vórtex.

Microscopicamente observa-se, na maioria dos corações, inflamação crônica de intensidade e extensão variáveis, localizada especialmente no miocárdio e no epicárdio; o endocárdio raramente mostra-se afetado.

A miocardite é geralmente focal (Fig. 3A), mas em aproximadamente $15 \%$, mostra-se zonal ou difusa. $O$ aspecto focal caracteriza-se por minúsculas áreas infiltradas por mononucleares (macrófagos, linfócitos e plasmócitos) em número variável, entre as fibras ou segmentos de fibras miocárdicas destruidas. Essas apresentam sinais degenerativos e/ou de necrose, tais como necrose hialina e miocitólise, invadidas pelo infiltrado mononuclear; em outros focos, as fibras destruídas são substituídas pelo estroma endomisial, colapsado e condensado, levando à formação de microcicatrizes.

$\mathrm{O}$ aspecto zonal da miocardite resulta da confluência de vários focos com as características citadas; nesse caso a destruição das fibras é mais extensa, assim como a cicatrização (Fig. 3B). Parte das fibras

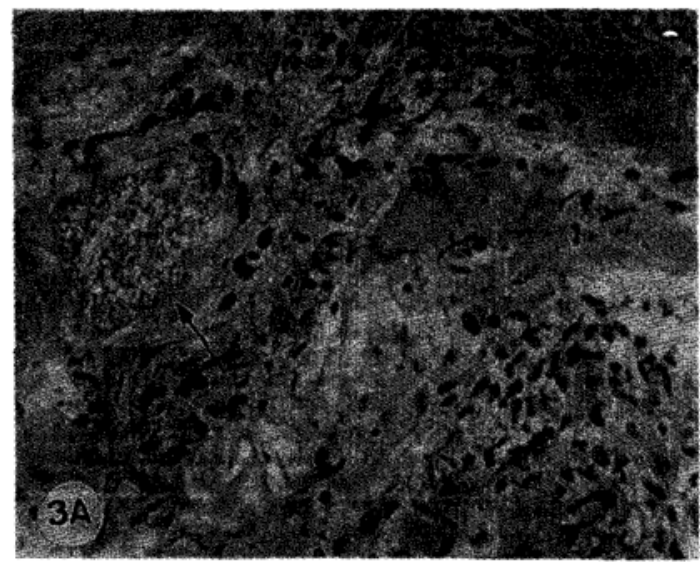

Fig. 3A - Miocardite crônica focal com infiltrado mononuclear; volumoso ninho de amastigotas do $\mathrm{T}$. cruzi (seta). Cepa Colombiana. HE $\times 100$.

Fig. 3B - Miocardite cronica com fibrose extensa, predominantemente endomisial, fenòmenos degenerativos, necrose e atrofia da maioria das miofibras; hipertrofia das demais. Cepa Y. HE $\times 100$.

Fig. 3C - Ganglionite atrial crônica; intenso infitrado de mononucleares e destruição da maioria dos neurönios. $H E \times 100$. não atingidas, pelos fenômenos degenerativos, mostram-se hipertróficas. Na forma difusa, extensas áreas de miocárdio, especialmente do ventrículo esquerdo, são atingidas; menos intensamente são acometidos o ventrículo direito, o átrio esquerdo e o átrio direito. Ninhos de amastigotas do $T$. cruz $i$ são encontrados em todos os grupos, embora em número escasso, tanto nas áreas de inflamação como fora delas, no interior de fibras aparentemente íntegras.

A epicardite caracteriza-se pela infiltração, focal ou zonal, de mononucleares (macrófagos, linfócitos e plasmócitos), localizada imediatamente abaixo do epitélio da serosa ou na profundidade do tecido gorduroso e nas estruturas anexas.

O tecido nervoso intracardíaco está acometido, na maioria absoluta dos ratos infectados, por infiltrado de mononucleares, de intensidade variável (ganglionite, periganglionite e neurite), com predominancia de linfócitos (Fig. 3C). Os neurônios dos gânglios exibem fenômenos degenerativos diversos, como degeneração hidrópica, cromatólise, hipercromasia e picnose nuclear. Alguns neurônios mostram-se substituídos por células-satélites (satelitose) com formação dos corpos de Nageotte. Em alguns corações, nos
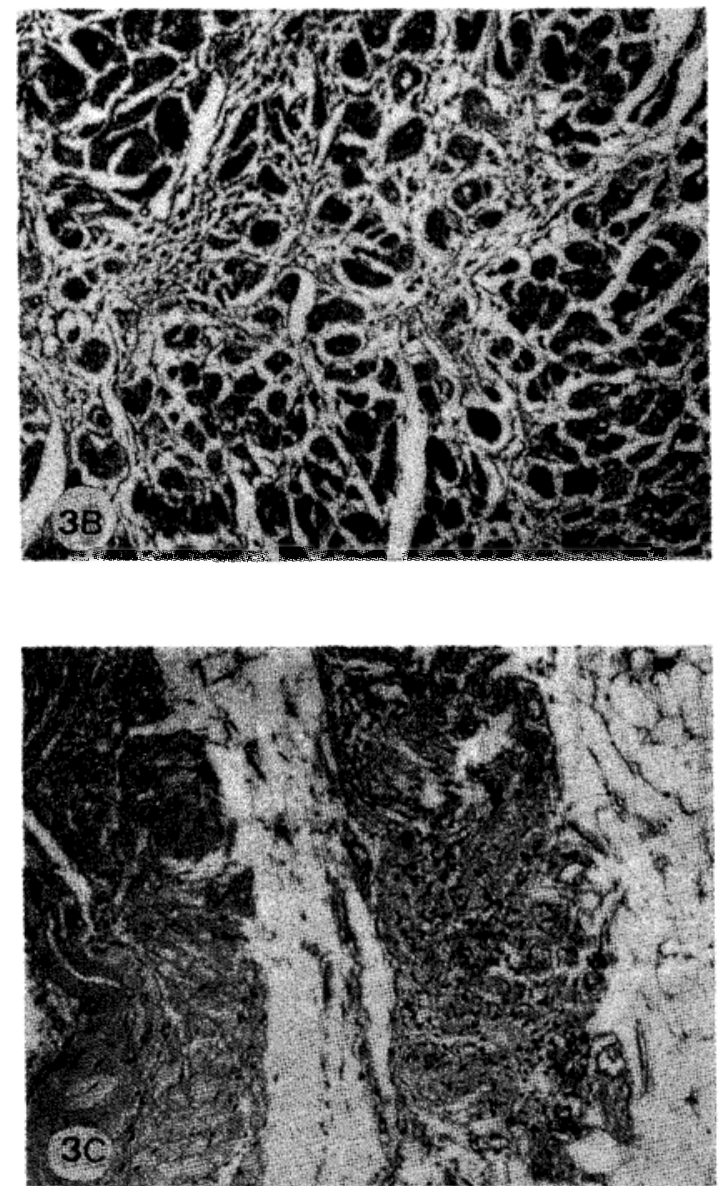
Chapadeiro E, Beraldo PSS, Jesus PC, Oliveira Jr. WP, Junqueira Jr. LF. Lesões cardíacas em ratos wistar inoculados com diferentes cepas do Trypanosoma cruzi. Revista da Sociedade Brasileira de Medicina Tropical 21: 95-103, Jul-Set, 1988

quais foi identificada estrutura paraganglionar, não se verificaram alterações histopatológicas.

No tecido excitocondutor observa-se o mesmo padrão de infiltrado inflamatório, porém em grau menor, caracterizado por pequenos acúmulos focais de mononucleares. Graus variáveis de fibrose e atrofia de fibras específicas, desse tecido, foram observados naqueles animais em que a miocardite adquiriu aspecto zonal ou difuso.

O endocárdio parietal, das diferentes câmaras, mostra somente raríssimos e minúsculos focos de infiltrado inflamatório mononuclear. Trombose organizada (antiga), do átrio esquerdo, foi encontrada com freqüência, particularmente, naqueles ratos inoculados com a cepa $Y$ do $T$. cruzi e mais raramente no ventrículo esquerdo. Nos casos em que ocorreram tromboses, esse folheto apresentava espessamentos fibrosos, focais ou difusos, com ou sem calcificação e/ou ossificação.

Nos animais com baixo inóculo, das cepas $\mathbf{Y}$ e 12SF não foram detectadas alterações histopatológicas, ou essas foram predominantemente de grau leve. Por outro lado, no subgrupo de ratos infectados com baixo inóculo da cepa Colombiana, foram observadas lesões histopatológicas no miocárdio atrioventricular, na inervação intrínseca e no tecido excitocondutor, de grau significantemente maior que nos subgrupos $\mathbf{Y}$ e SF $(P<0,05)$. Nos subgrupos de ratos infectados com os inóculos médio e alto, das cepas $Y$ e 12SF, e naqueles com o inóculo médio, da cepa Colombiana, as lesões histopatológicas, das diversas estruturas examinadas, foram de graus semelhantes entre si, porém mais elevados em relação ao grup controle $(P<0,05)$. Somente a cepa Colombiana do $T$. cruzi foi capaz de induzir lesões cardíacas crônicas com baixo inóculo (2-3000 parasitos/g).

Considerando cada subgrupo de ratos chagásicos e as diversas estruturas cardiacas examinadas, 0 máximo "score" médio atingido foi de 3,5 $\pm 1,9$, em uma escala de possibilidade até 6 . Isto significa que o acometimento crônico do coração, nos ratos chagásicos, foi usualmente de grau leve a moderado. Alguns animais, entretanto, inoculados com quaisquer das cepas, exibiram graves lesões cardíacas, particularmente de inervação intrínseca e do miocárdio atrial ou ventricular.

Analisando, do ponto de vista percentual, a histopatologia do coração, de animais que exibiram lesões cardíacas de qualquer grau, observa-se (Fig. 4) que quanto maior o grau do inoculo aplicado, das cepas $Y$ e 12SF, maior o número de animais que exibiram lesões histopatológicas das diversas estruturas do coração. $O$ percentual desses animais infectados com o baixo inóculo variou de $6 \%$ (ratos com miocardite atrial) a $59 \%$ (ratos com pericardite). Nenhum dos ratos infectados, com o baixo inóculo da cepa SF, exibiu miocardite ventricular ou lesão da

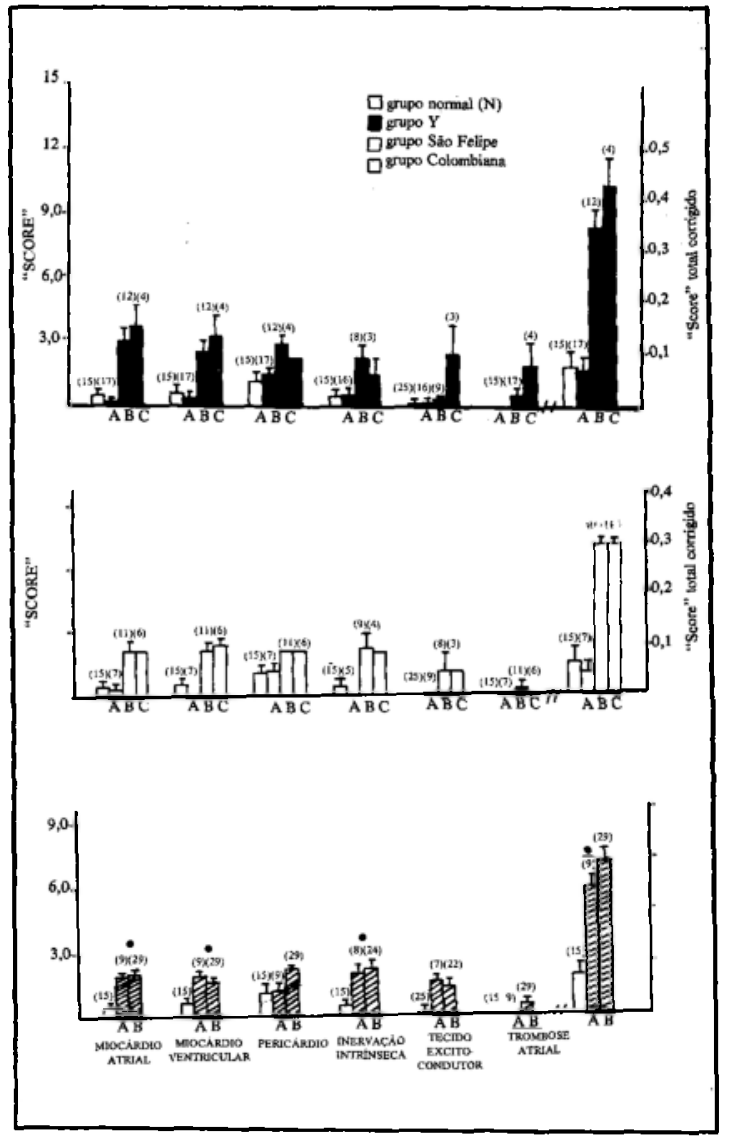

Fig. 4 - Grau de alteração histopatológica, expressa pelo "score" médio e pelo "score" total corrigido, nas diversas estruturas do coraçâo, e manifestação de trombose atrial, exibidos pelos subgrupos de ratos chagásicos e pelo Grupo-controle, sacrificados no 8.0 mês de seguimento (fase crónica da infecção).

$p<0,05$ (Subgrupo COL/baixo inóculo x Grupocontrole e Subgrupos Y e SF/Baixo inóculo análise de variância, com teste de NewmanKeuls).

Nos subgrupos de ratos infectados com os inóculos médio e alto das cepas Ye SF e naquele com o inóculo médio da cepa COL, o grau de lesão histopatológica foi semelhante, mas, mostrou-se elevado em relação ao Grupo-controle $(p<0,05-$ análise de variância, com teste de NewmanKeuls).

$A=$ baixo inóculo (2-3 000 parasitos $/ g$ );

$B=$ médio inóculo (4-5 000 parasitos $/ g$ );

$C=$ alto inóculo (6 000 parasitos $/ g$ ).

( ) Número de ratos nos quais a estrutura foi yisualizada. 
inervação intríseca e do tecido excitocondutor. $\mathrm{O}$ percentual dos animais com lesões cardíacas, infectados com médio e alto inóculo dessas cepas, variou de $12 \%$ (ratos com lesão do tecido excitocondutor) a $100 \%$ (ratos com lesão das demais estruturas). No caso da cepa Colombiana, o percentual de animais infectados, com os inóculos baixo e médio que exibiram lesões cardíacas, variou de $45 \%$ (ratos com lesão do tecido excitocondutor) a $96 \%$ (ratos com pericardite). Portanto, um elevado percentual de ratos infectados, com os inóculos médio e alto das cepas $Y$ e 12SF e, com os inóculos baixo e médio da cepa Colombiana, exibiu lesões nas diversas estruturas cardíacas examinadas.

\section{COMENTÁRIOS}

Nossos achados confirmam, de um modo geral, as observações anteriores 126891214161718 de que o rato albino Wistal é suscetivel à infecção por diferentes cepas do Trypanosoma cruzi e responde com uma fase inicial (aguda) e uma tardia (crônica), da mesma maneira que o homem e outras espécies animais. Portanto, o rato albino não parece um animal altamente resistente à infecção chagásica como admitem alguns, nem tampouco um modelo no qual a infecção adota caráter imprevisivel ${ }^{3} 56$. Contrariamente às opiniões de Dias ${ }^{9}$ e Pizzi e cols ${ }^{13}$, a resistência do rato ao Trypanosoma cruzi está na dependência do inóculo empregado. Nas condições experimentais deste trabalho, os animais foram mais sensiveis às cepas Colombiana, Y e 12SF, respectivamente. De outro lado, a infecção, medida por vários parâmetros, teve comportamento similar e as lesões foram quase constantes, guardando estreita relação com o inóculo utilizado. De fato, na base de um sistema de "score" foi possivel correlacionar, precisamente, o grau das lesões histológicas não só com as alterações eletrocardiográficas e da função autonômica, mas também com outros aspectos gerais relativos à infecção 4 .

Nos animais que morreram na fase inicial da infecção, observou-se, como no homem ${ }^{7}$ e em outros modelos experimentais ${ }^{5}$, cardite aguda, de intensidade e extensão variáveis, atingindo as diversas estruturas do coração com parasitismo variável, mas sempre presente. Além dessas lesões apareceu, na maioria dos ratos, trombose do átrio esquerdo, a qual, provavelmente, resultou da insuficiência cardíaca aguda; é claro que não se pode excluir a alteração do endocárdio atrial, em conseqüência da própria inflamação, como causa da trombose.

$\mathrm{Na}$ fase tardia (crônica) da infecção, as lesões inflamatórias do coração variaram entre focal, zonal e/ou difusa, acometendo principalmente o miocárdio dos ventrículos e o sistema nervoso intracardíaco com conseqüente destruição neuronal (desnervação) e, em menor grau, o sistema excitocondutor. $O$ aspecto focal da inflamação no miocárdio é semelhante àquele encontrado no coração dos portadores da forma indeterminada da doença ou daqueles chagásicos que falecem subitamente, de modo inesperado 1011 . De outro lado, o aspecto da miocardite crônica, zonal ou difusa, é semelhante ao quadro da forma humana que se manifesta por arritmias ou insuficiência cardíaca congestiva ${ }^{3}$. Embora, do ponto de vista clínico, não tenha sido possível evidenciar sinais de insuficiência cardíaca, nesses animais ${ }^{4}$, foram observadas, com freqüência, tromboses recentes e antigas (organizadas) no átrio e ventrículo esquerdos. Em alguns corações a dilatação do ventrículo esquerdo, na região do vórtex, sugere a ocorrência de lesão semelhante à cardiopatia humana ${ }^{15}$.

\section{SUMMARY}

Wistar albino rats infected intraperitonially with different inocula of $Y, 12 S F$ and Colombian strains of Trypanosoma cruzi developed high parasitemia, which lowered by the end of the 6th week; mortality varied according with the strain and inoculum.

Acute carditis like that occurring in the human, was found in all animals killed or dying spontaneously. The surviving rats were sacrificed in the 8 th month of infection. They presented active chronic carditis involving especially the myocardium and the intracardiac nervous system. In most of the animals the myocardial inflammation was focal. In approximately $15 \%$ it was zonal or difuse with fibrosis associated with muscle cell hypertrophy. Recent and old thrombosis as well as left ventricular apical dilation occurred in about $20 \%$ of those animals.

The authors confirm that the albino rat is susceptible to chagasic infection and conclude that it represents a useful model for the study of cardiac manifestations of the human disease.

Key-words: Chagas' infection. Experimental Chagas' disease. Experimental Chagasic cardiopathy.

\section{AGRADECIMENTOS}

Os AA. agradecem às senhoritas Valéria Ramos, Luzia Helena G. dos Santos pela parte técnica e Maria Laura P. Rodrigues pela confecção do manuscrito.

\section{REFERÊNCIAS BIBLIOGRÁFICAS}

1. Alcântara FG. Sistema neuro-vegetativo do coração na moléstia de Chagas experimental. Revista Goiana de Medicina 7:111-126, 1961. 
2. Alcântara FG. Moléstia de Chagas experimental (iManifestações viscerais). O Hospital 66:625-633, 1964.

3. Andrade ZA, Andrade SG. Patologia. In: Brener Z, Andrade ZA (ed) Trypanosoma cruzi e Doença de Chagas, Guanabara Koogan, Rio de Janeiro, p. 199-248, 1979.

4. Beraldo PSS. Sobre a infecção chagásica experimental no rato. Estudo eletrocardiográfico seriado e funcional autonômico do coração correlacionado à histopatologia. Tese de Mestrado, Universidade de Brasilia, Brasilia, 1987.

5. Brener Z, Ramirez LE. Modelo crônico da doença de Chagas experimental. In: Cançado JR, Chuster M(ed) Cardiopatia Chagásica, Fundação Carlos Chagas, Belo Horizonte, p. 23-28, 1985.

6. Castagnino HE, Thompson AC. Cardiopatia chagásica experimental. In: Cardiopatia Chagásica (ed) Kapelusz, Buenos Aires, p. 300-301. 1980.

7. Chapadeiro E, Tafuri WL, Lopes ER. Anatomia Patológica da cardiopatia aguda. In: Cançado JR, Chuster M (ed) Cardiopatia Chagásica, Fundação Carlos Chagas, Belo Horizonte, p. 29-32, 1985.

8. Costa RB. Hipertrofia cardíaca experimental em ratos chagásicos e ratos atropinizados. Revista do Instituto de Medicina Tropical de São Paulo 6:17-27, 1964.

9. Dias E. Estudo sobre o Schizotrypanum cruzi. Memórias do Instituto Oswaldo Cruz 28:1-1 10, 1934.

10. Lopes ER, Chapadeiro E, Almeida HO, Rocha A. Contribuição ao estudo da anatomia patológica dos corações de chagásicos falecidos subitamente. Revista da Sociedade Brasileira de Medicina Tropical 9:269282,1975

11. Lopes ER, Chapadeiro E, Rocha A. Anatomia patológica do coração na forma indeterminada. In: Cançado
JR, Chuster M (ed) Cardiopatia chagásica, Fundação Carlos Chagas, Belo Horizonte, p. 33-40, 1985.

12. Oliveira JSM. Cardiopatia chagásica experimental. Revista Goiana de Medicina 15:77-133, 1969.

13. Pizzi $T$, Rubio MD, Knierim FT. Contribución al conocimiento de los mecanismos imunitarios en la enfermedad de Chagas experimental en la rata. Boletin de Informaciones Parasitarias Chilenas 8:66-72, 1953.

14. Postan M, McDaniel JP, Dvorak JA. Comparative studies of the infection of Lewis rats with four Trypanosoma cruzi clones. Transactions of the Royal Society of Tropical Medicine and Hygiene 81:415-419, 1987.

15. Raso P, Chapadeiro E, Tafuri WL, Lopes ER, Rocha A. Anatomia patológica da cardiopatia crônica. In: Cançado JR, Chuster M (ed) Cardiopatia Chagásica, Fundação Carlos Chagas, Belo Horizonte, p. 41-53, 1985.

16. Revelli SS, Amerio N, Moreno HS, Valenti JL, Balbarrey H, Morini JC. Enfermedad de Chagas cronica en la rata. Características sorologicas, eletrocardiograficas y histopatologicas. Medicina (Buenos Aires) 40 (Supl. 1): 69-76, 1980.

17. Scorza C, Scorza JV. Acute myocarditis in rats inoculated with Trypanosoma cruzi: study of animals sacrificed between the fourth and twenty-ninth days after infection. Revista do Instituto de Medicina Tropical de São Paulo 14:171-177, 1972.

18. Vichi FL. Avaliação quantitativa do parasitismo no coração, vasos da base e coronária do rato, na fase aguda da moléstia de Chagas. Revista do Instituto de Medicina Tropical de São Paulo 6:292-296, 1964.

19. World Health Organization. Report of the scientific working group on the development and evaluation of animal models for Chagas' disease. Geneve, p. 10 , 1984. 